\title{
Square-free words with square-free self-shuffles
}

\author{
James D. Currie \& Kalle Saari \\ Department of Mathematics and Statistics \\ University of Winnipeg \\ 515 Portage Avenue \\ Winnipeg, MB R3B 2E9, Canada \\ j.currie@uwinnipeg.ca, kasaar2@gmail.com
}

Submitted: Jul 27, 2013; Accepted: Jan 5, 2014; Published: Jan 12, 2014

\begin{abstract}
We answer a question of Harju: For every $n \geqslant 3$ there is a square-free ternary word of length $n$ with a square-free self-shuffle.
\end{abstract}

\section{Introduction}

Shuffles of words are natural objects of study in combinatorics on words, and a variety of interesting problems have been posed. (See [5], for example.) Recently, self-shuffles of words have been studied. (See, for example $[7,8]$ which independently show that it is NP-complete to decide whether a finite word can be written as a self-shuffle.) If a word $w$ is factored as

$$
w=\Pi a_{i}=\Pi b_{i}
$$

where $a_{i}, b_{i} \neq \epsilon$, then we call

$$
\Pi\left(a_{i} b_{i}\right)
$$

a self-shuffle of $w$. For example, letting $w=01101001, a_{1}=011, a_{2}=01, a_{3}=001$, $b_{1}=01, b_{2}=1010, a_{3}=01$, we get the self-shuffle of $w$

$$
011 \underline{0101} \underline{101000101} \text {. }
$$

(Here the $b_{i}$ have been underlined for ease of reading.) The notion of a self-shuffle equally applies to infinite words, and in [3] it is shown that the Fibonacci word has a self-shuffle which is equal to the Fibonacci word; similarly, it is shown that the Thue-Morse word is equal to one of its self-shuffles.

The recent note of Harju [4] poses this problem:

Problem 1.1. For every $n \geqslant 3$ is there a square-free word of length $n$ with a square-free self-shuffle? 
In this paper we answer this question in the affirmative; in fact the desired square-free words can be found over a ternary alphabet. In what follows, we freely use the usual notions of combinatorics on words. A standard reference is [6].

\section{Long finite square-free words with square-free self- shuffles}

Consider a square-free word $u \in\{0,1,2\}^{*}$ such that neither of 010 and 212 is a factor of $u$, and $u$ is of the form

$$
u=0120 w_{0} \Pi_{i=1}^{m}\left(a w_{i}\right) 2012
$$

where $m \in\{0,1,2,3\}$, the $w_{i} \in\{0,1,2\}^{*}$ and $a=2021020$. We will show later that such words $u$ of length $n$ exist for all large enough $n \equiv 3(\bmod 4)$.

Let $b=2021201020$. Let $\bar{u}$ be the word

$$
\bar{u}=0120 w_{0} \Pi_{i=1}^{m}\left(b w_{i}\right) 2012 .
$$

The longest prefix of $b$ not containing 212 is 2021, which is also a prefix of $a$. The longest suffix of $b$ not containing 010 is 1020 , which is also a suffix of $a$. It follows that any factor of $\bar{u}$ not containing 010 or 212 is itself a factor of $u$.

Now consider the self-shuffle $w$ of $\bar{u}$ given by

$$
w=\bar{u} 2^{-1} 020^{-1} \bar{u}=0120 w_{0} \Pi_{i=1}^{m}\left(b w_{i}\right) 20102120 w_{0} \Pi_{i=1}^{m}\left(b w_{i}\right) 2012 .
$$

The prefix of $w$ of length $|\bar{u}|-1$ is a prefix of $\bar{u}$, while the prefix of $w$ of length $|\bar{u}|$ has suffix 010. The suffix of $w$ of length $|\bar{u}|-1$ is a suffix of $\bar{u}$, while the suffix of $w$ of length $|\bar{u}|$ has prefix 212. It follows that the only factors of $w$ not containing either 010 or 212 must themselves be factors either of $\bar{u}$ or of 1021; by the previous paragraph, they are factors of $u$ or of 1021, and in particular are square-free. At this point we will mention that many arguments can be shortened by noting that the definitions of $a, b, u, \bar{u}$ and $w$ are invariant under the operation combining reversal with the substitution $k \rightarrow 2-k$

on each letter. Particular words $u$ and $\bar{u}$ need not be invariant under this operation, but they are sent to words of the same form.

Lemma 2.1. Consider a square-free word $u$ of the form (1) and let $\bar{u}$ and $w$ be defined as in (2) and (3). Fix $j, 0 \leqslant j \leqslant m$, and let a word $U$ be obtained from $\bar{u}$ by replacing some $j$ occurrences of b by $a$. Let $W$ be obtained from $w$ by making the analogous replacements. Thus $W=U 2^{-1} 020^{-1} U$. Then $U$ and $W$ are square-free. In particular, words $\bar{u}$ and $w$ are square-free.

Proof. Suppose not. Consider a word $U$ obtained from $\bar{u}$ such that one of $U$ and $W$ contains a square, and such that $m-j$ is as small as possible.

We deal first with the case where $m-j=0$. In this case, $U=u$ is automatically square-free, and any factor of $W=w$ not containing 010 or 212 is square-free. Let $y y$ be a factor of $W=w, y \neq \epsilon$. Thus, one of 010 or 212 is a factor of $y y$. 
If $|y|_{010} \geqslant 1$ then $|y y|_{010} \geqslant 2|y|_{010} \geqslant 2$; however, $|y y|_{010} \leqslant|w|_{010}=1$. It follows that in fact $|y|_{010}=0$. Similarly, $|y|_{212}=0$. Now if 010212 is a factor of $y y$, then depending on how 010212 is distributed between the two copies of $y$, at least one of 010 and 212 must be a factor of $y$. This is impossible, so that 010212 is not a factor of $y y$. It follows that $y y$ must be a factor of one of $0120 w_{0} \Pi_{i=1}^{m}\left(a w_{i}\right) 201021$ and $102120 w_{0} \Pi_{i=1}^{m}\left(a w_{i}\right) 2012$. (These are, respectively, the longest prefix and the longest suffix of $w$ not containing 010212.)

Suppose then that $y y$ is a factor of $0120 w_{0} \Pi_{i=1}^{m}\left(a w_{i}\right) 201021$. (The other case is similar.) Then 212 is not a factor of yy, forcing 010 to be a factor of yy. However, 010 must not be a factor of $y$, so that, depending on how 010 is split between copies of $y$, we can write $y=p 0=10 s$ or $y=p 01=0 s$, where $s$ must be a prefix of 21, $p$ a suffix of $0120 w_{0} \Pi_{i=1}^{m}\left(a w_{i}\right) 2$. However, $y=p 0=10 s$ is impossible; if $s \neq \epsilon$, then the word on the right-hand side of this equation ends in 1 or 2 , while the left-hand word ends in 0 ; if $s=\epsilon$, $p=1$, which is not a suffix of $0120 w_{0} \Pi_{i=1}^{m}\left(a w_{i}\right) 2$. Again, $y=p 01=0 s$ forces $s=21$, since the left-hand word ends in 1; however $p 01$ doesn't end in 21.

This shows that $m-j=0$ is impossible. We now have $m-j>0$, so that multiple copies of 010 and 212 appear in $W$. It will be useful to work out the distances between occurrences of 010 , that is, the minimum value of $|010 v|$ such that $010 v 010$ is a factor of $W$. From the definition of $W$, any word $010 v$ such that $010 v 010$ is a factor of $W$ is at least as long as a word of the form $01020 w_{i} 20212,01020 w_{m} 2$ or $0102120 w_{0} 20212$. From the definition of $u$, factor $020 w_{m} 2012$ of $a w_{m} 2012$ is square-free, and doesn't contain 010 or 212. This implies that $w_{m}$ has prefix 1 and suffix 0 . However, $w_{m} \neq 10$ or else $a w_{m}$ would contain $0 w_{m}$, which starts with 010. In particular $\left|w_{m}\right| \geqslant 3$, and $|010 v| \geqslant$ $|01020|+3+|2|=9$, and $|v| \geqslant 6$. From (3) we see that this argument also guarantees that any factor $010 v 010$ of $U$ will also have $|v| \geqslant 6$.

Suppose $y y$ is a square in $W$ or in $U, y \neq \epsilon$. Suppose now that $|y|_{010}>0$. Note that 010 occurs in $W$ or $U$ in one of only two possible contexts: either $20212 \underline{01020}$ or $02 \underline{0102120 .}$ Observing the 3 characters to the left of an occurrence of 010 is enough to identify this context. If the 3-character string to the left is 212, then the context is 2021201020; if the 3-character string is not 212, then the context is 020102120 (since $w_{m}$ ends in 0.) Similarly, examining the three characters to the right of an occurrence of 010 establishes its local context. Let us write $y=p 010 s$. Then $010 s p 010$ is a factor of $W$ or $U$ and $|s p| \geqslant 6$, so that at least one of $|p|,|s| \geqslant 3$. This establishes the local context of a certain occurrence of 010 in both copies of $y$, and these contexts must be the same. Since the local context 20102120 only occurs exactly once in $W$, and never in $U$, both local contexts of 010 in $y$ are as a factor of $b$. Similarly, if $|y|_{212}>0$, then 212 appears in a local context coming from $b$. In fact, this argument shows that $|y y|_{010212}=0$; if $|y y|_{010212}=1$, then at least half of the occurrence of 010212 lies inside one copy of $y$, so that an occurrence of 010 or of 212 in $y$ comes from 20102120, which is impossible. Therefore, if $y y$ is a factor of $W$, we conclude that $y y$ is a factor of one of $U 2^{-1}$ and $0^{-1} U$, the longest prefix and suffix, respectively, of $W$ not containing a 010 or 212 coming from 20102120; however, this prefix and suffix are themselves factors of $W$, so that we see that $y y$ must be a factor of $U$.

We have shown that any occurrences of 010 in $y$ arise as factors of $b$. Write $b^{\prime}=20212$, 
$b^{\prime \prime}=20$, so that $b=b^{\prime} 010 b^{\prime \prime}$. We are thus saying that any occurrence of 010 in $y$ is preceded (in W) by $b^{\prime}$ and followed by $b^{\prime \prime}$. Suppose $|y|_{010} \geqslant 1$. Write $y=p 010 s$. Suppose $|y|_{b}=0$. Then either $|p|<\left|b^{\prime}\right|$ or $|s|<\left|b^{\prime \prime}\right|$. If $|p|<\left|b^{\prime}\right|$, write $W=x y y z$. Then $b^{\prime}$ must be a suffix of both $x p$ and $y p$. Let $\sigma$ be the common suffix of $x$ and $y$ such that $\sigma p=b^{\prime}$. Replacing $y$ by $\sigma y \sigma^{-1}$, we have a square $y y$ in $W$ such that $|y|_{b}=1$. The case where $|s|<\left|b^{\prime \prime}\right|$ is similar; in either case, if $|y|_{010}>0$, then adjusting $y y$ cyclically if necessary, we can assume that $|y|_{b}>0$. Now, replacing $b$ 's in $y$ (and hence in $U$ ) by $a$ 's yields a square in a word of the form of $U$, with the same $m$, but larger $j$. This contradicts the minimality of $m-j$.

From now on, we can assume that $|y|_{010},|y|_{212}=0$ and $y y$ is a factor of $U$. If $|y y|_{212010}>0$, then depending on how 212010 is split between the copies of $y$, at least one of $|y|_{010}$ and $|y|_{212}$ is non-zero. We conclude that $|y y|_{212010}=0$. By the same argument as earlier, any factors of $U$ not containing 010 or 212 are square-free. It follows that at least one of $|y y|_{010}$ and $|y y|_{212}$ is non-zero. Without loss of generality (up to reversal and 2-complementation) suppose that $|y y|_{010}>0$. Since $|y|_{010}=0$, we must be able to write $y=p 0=10 s$ or $y=p 01=0 s$ where $p$ is a suffix of 12 (since $|y|_{212}=0$.) If $y=p 0=10 s$, each of $p=12,2, \epsilon$ is seen to be impossible. If $y=p 01=0 s$, then $p$ begins with 0 , which is also impossible.

We conclude that $W$ and $U$, and hence $w$ and $\bar{u}$, cannot contain a non-empty square yy.

As promised, we now show that words of the form $u=0120 w_{0} \Pi_{i=1}^{m}\left(a w_{i}\right) 2102$ of length $n$ exist for all large enough $n \equiv 3(\bmod 4)$.

The Thue-Morse word is the sequence $\mathbf{t}=\mu^{\omega}(0)$ where $\mu(0)=01, \mu(1)=10$. Word $\mathbf{t}$ is well-known to be overlap-free. From the definition of $\mathbf{t}$ it is clear that $\mathbf{t} \in\{01,10\}^{*}$. On occasion it is useful to add 'bar lines' to a factor of $\mathbf{t}$ indicating the parsing of $\mathbf{t}$ in terms of 01 and 10. These bar lines always split any occurrence of 00 or 11 ; viz, $0 \mid 0$ or $1 \mid 1$, not $|00|$ or $|11|$. It is proved in [1, Lemma 4] that $\mathbf{t}$ contains a factor of the form $10 x 01$ of every length greater than or equal to 6 .

Consider the word s obtained from the Thue-Morse word by counting 1's between subsequent 0's. Thus if we write

$$
\mathbf{t}=\Pi 01^{s_{i}}
$$

then

$$
\mathbf{s}=\Pi s_{i} .
$$

It is well-known that $\mathbf{s}$ is square-free. It is also well-known and easily verified that neither of 010 and 212 is a factor of $\mathbf{s}$.

Lemma 2.2. Word $\mathbf{s}$ contains a factor of the form $0120 x 2012$ of every length $n \equiv 3$ (mod 4), $n \geqslant 23$.

Proof. A factor of $\mathbf{s}$ of the form $z=0120 x 2012$ corresponds to a factor

$$
v=00101100 y 0110010110
$$


of t. For clarity, add 'bar lines' to $v$ :

$$
v=0|01| 01|10| 0 y 01|10| 01|01| 10 \text {. }
$$

The number of 0's in $v$ is one more than the length of $z$, giving $|z|=|v|_{0}-1=(|v|-1) / 2$.

s contains a factor of form $z$ of length $k$

$\Rightarrow \mathbf{t}$ contains a factor of form $v$ of length $2 k+1$

$\Rightarrow \mathbf{t}$ contains a factor of form $10|01| 0 y^{\prime} 0|10| 01$ of length $k+1$

$\Rightarrow \quad k$ is odd and $\mathbf{t}$ contains a factor of form $10\left|0 y^{\prime \prime} 1\right| 10$ of length $(k+1) / 2$

$\Rightarrow \quad(k+3) / 2$ is even and $\mathbf{t}$ contains a factor of form $10 \hat{y} 01$ of length $(k+1) / 4$

The result follows.

The words $z$ of the last lemma begin and end in the form desired for $u$. We will now show when $z$ is long enough, word $a=2021020$ is a factor of $z$ at least 5 times. Although the first and last occurrences of $a$ may overlap with the prefix 0120 or suffix 2012 of $z$, there will be at least three other occurrences of $a$ in $z$, so that for any $m \in\{0,1,2,3\}$ we can write $z$ in the form

$$
z=0120 w_{0} \Pi_{i=1}^{m}\left(a w_{i}\right) 2012
$$

as desired.

Lemma 2.3. Suppose that $02102 v 02102$ is a factor of $\mathbf{s}$, but that 02102 is not a factor of 2102v0210. Then $|02102 v 02102| \leqslant 41$.

Proof. A factor 02102 of s corresponds to a factor $0|01| 10|10| 01|10|$ of t. Such factors of $\mathbf{t}$ occur precisely in the context $01|10| 01|10| 10|01| 10 \mid 01=\mu^{2}(0011)$. A factor $02102 v 01202$ of $\mathbf{s}$ such that 02102 is not a factor of $2102 v 0210$ corresponds to a factor $(011)^{-1} \mu^{2}(0011 u 0011)(01)^{-1}$ of $\mathbf{t}$ which does not contain 0011 as an internal factor. Word $\mathbf{t}$ is concatenated from $\mu^{4}(0)=0110100110010110$ and $\mu^{4}(1)=1001011001101001$, and each of these contains a factor 0011. In addition, concatenating suffix 0 and prefix 011 of $\mu^{4}(0)$ produces a factor 0011; so does concatenating suffix 001 and prefix 1 of $\mu^{4}(1)$. We therefore see that the longest factor $0011 u 0011$ of $\mathbf{t}$ with no internal 0011 is the word $00110010110 \mid 10010110011$, of length 22 .

We have determined that $0210 v 0210$ corresponds to a factor

$$
z=(011)^{-1} \mu^{2}(0011 u 0011)(01)^{-1}
$$

of $\mathbf{t}$ where $|0011 u 0011| \leqslant 22$. Because $\mathbf{s}$ is obtained from $\mathbf{t}$ by counting 0 's and $z$ begins and ends with 0 ,

$$
|02102 v 02102|=|z|_{0}-1
$$


Every second letter of $\mu^{2}(0011 u 0011)$ is a 0 , so that

$$
\begin{aligned}
|z|_{0} & =\left|\mu^{2}(0011 u 0011)\right|_{0}-|011|_{0}-|01|_{0} \\
& =\left|\mu^{2}(0011 u 0011)\right| / 2-2 \\
& =2|0011 u 0011|-2 \\
& \leqslant 2(22)-2 \\
& =42 .
\end{aligned}
$$

We conclude that $|02102 v 02102| \leqslant 41$.

Corollary 2.4. Any factor of $\mathrm{s}$ of length 40 contains 02102 as a factor.

Corollary 2.5. Any factor of $\mathbf{s}$ of length $4^{2}$ contains $a=2021020$ as a factor.

Proof. The word 02102 cannot be preceded by 1 or 0 in $s$; It follows that 02102 can only be preceded by 2 in s. Similarly, 02102 is only followed by 0 . Any length 42 factor $v$ of s contains 02102. Extending $v$ before and after by one character then forces $a$ to be a factor.

Corollary 2.6. Any factor $z$ of $\mathbf{s}$ of the form $0120 x 2012$ of length at least 134 can be written in the form

$$
z=0120 w_{0} \Pi_{i=1}^{m}\left(a w_{i}\right) 2012 .
$$

Proof. Since $134=|0120|+3(42)+|2012|$, the result follows by the previous Corollary.

Theorem 2.7. For every $n \geqslant 143$ there is a square-free word $u \in\{0,1,2\}^{*}$ of length $n$ which permits a square-free self-shuffle.

Proof. We note that $|b|-|a|=3$. Given $n \geqslant 143$, let $m$ be least such that $n-3 m \equiv 3$ $(\bmod 4)$. We have $|n-3 m| \geqslant 143-3(3)=134$. By Lemma 2.2 there is a factor $u$ of $\mathbf{s}$ of the form $u=0120 x 2012,|z|=n-3 m$. By Lemma 2.6, word $u$ has the form

$$
u=0120 w_{0} \Pi_{i=1}^{m}\left(a w_{i}\right) 2012 .
$$

Letting

$$
\bar{u}=0120 w_{0} \Pi_{i=1}^{m}\left(a w_{i}\right) 2012
$$

gives a word $\bar{u}$ of length $n$, and by Lemma 2.1, both $\bar{u}$ and the self-shuffle

$$
w=\bar{u} 2^{-1} 020^{-1} \bar{u}
$$

of $\bar{u}$ are square-free. 


\section{Short square-free words with square-free self-shuffles}

It is well-known that $\mathbf{s}$ is the fixed point of $2 \mapsto 210,1 \mapsto 20,0 \mapsto 1$.

Lemma 3.1. For every $n$ with $3 \leqslant n \leqslant 200$, there exists a ternary square-free word with a self-shuffle that is also square-free.

Proof. The following claims can be checked computationally ${ }^{1}$.

For each $n$ with $29 \leqslant n \leqslant 200$, s has a factor $w$ of length $|w|=n$ such that the shuffle $p_{1} p_{2} s_{1} s_{2}$ is square-free, where $w=p_{1} s_{1}=p_{2} s_{2}$. Furthermore, the lengths of $s_{1}$ and $p_{2}$ can be restricted to satisfy $1 \leqslant\left|s_{2}\right|,\left|p_{1}\right| \leqslant 3$.

For each $n$ with $3 \leqslant n \leqslant 28$ except for $n=10$, there exist a ternary square-free word $w$ with a square-free self-shuffle $p_{1} p_{2} s_{1} s_{2}$ as above. The difference with the above is that we cannot always take $w$ to be a factor of $\mathbf{s}$ and the lengths of $s_{1}$ and $p_{2}$ cannot be restricted as much.

Finally, for $n=10$, one can take the square-free word $w=0102120102$, which has the following square-free self-shuffle:

$0102 \underline{012} \underline{1020102120102 .}$.

Combining this with the result of the previous section solves Harju's problem:

Theorem 3.2. For every $n \geqslant 3$, there exists a ternary square-free word of length $n$ having a square-free self-shuffle.

\section{References}

[1] A. Aberkane \& J. D. Currie, There exist binary circular $5 / 2+$ power free words of every length, Elec. J. Comb. 11 (2004), R10.

[2] F.-J. Brandenburg, Uniformly growing $k$-th power-free homomorphisms, Theoret. Comput. Sci. 23 (1983), 69-82.

[3] Émilie Charlier, Teturo Kamae, Svetlana Puzynina, \& Luca Q. Zamboni. Selfshuffling words, arXiv: 1302.3844 (2013)

[4] T. Harju, A note on square-free shuffles of words, LNCS, WORDS 2013. To appear.

[5] D. Henshall, N. Rampersad, \& J. Shallit, Shuffling and unshuffling, Bull. EATCS, 107 (2012), 131-142.

[6] M. Lothaire, Combinatorics on Words, Encyclopedia of Mathematics and its Applications 17, Addison-Wesley, Reading, 1983.

[7] S. Buss, M. Soltys, Unshuffling a square is NP-hard, arXiv: 1211.7161 (2013).

[8] R. Rizzi and S. Vialette. On recognizing words that are squares for the shuffle product, CSR 2013, LNCS 7913 (2013), 235-245.

\footnotetext{
${ }^{1}$ An IPython notebook showing these computations can be found in http://users.utu.fi/kasaar/ square-free_shuffles.ipynb
} 